\title{
The Influence of Process Conditions on the Chemical Composition of Pine Wood Catalytic Pyrolysis Oils
}

\author{
J. Pereira, ${ }^{1}$ F. A. Agblevor, ${ }^{2}$ and S. H. Beis ${ }^{1}$ \\ ${ }^{1}$ Department of Biological Systems Engineering, Virginia Polytechnic Institute and State University, Blacksburg, VA 24061, USA \\ ${ }^{2}$ Department of Biological Engineering, Utah State University, Logan, UT 84341, USA
}

Correspondence should be addressed to F. A. Agblevor, foster.agblevor@usu.edu

Received 25 October 2012; Accepted 19 November 2012

Academic Editors: C. Koroneos and W. E. Mabee

Copyright ( $) 2012$ J. Pereira et al. This is an open access article distributed under the Creative Commons Attribution License, which permits unrestricted use, distribution, and reproduction in any medium, provided the original work is properly cited.

\begin{abstract}
Pine wood samples were used as model feedstock to study the properties of catalytic fast pyrolysis oils. The influence of two commercial zeolite catalysts (BASF and SudChem) and pretreatment of the pine wood with sodium hydroxide on pyrolysis products were investigated. The pyrolysis oils were first fractionated using column chromatography and characterized using GCMS. Long chain aliphatic hydrocarbons, levoglucosan, aldehydes and ketones, guaiacols/syringols, and benzenediols were the major compounds identified in the pyrolysis oils. The catalytic pyrolysis increased the polycyclic hydrocarbons fraction. Significant decreases in phthalate derivatives using SudChem and long chain aliphatics using BASF catalyst were observed. Significant amounts of aromatic heterocyclic hydrocarbons and benzene derivatives were formed, respectively, using BASF and SudChem catalysts. Guaiacyl/syringyl and benzenediols derivatives were partly suppressed by the zeolite catalysts, while the sodium hydroxide treatment enriched phenolic derivatives. Zeolite catalyst and sodium hydroxide were employed together; they showed different results for each catalyst.
\end{abstract}

\section{Introduction}

Biomass is a renewable and alternative resource that can be pyrolyzed to produce fuels and chemicals. Pyrolysis oils can potentially be substituted for fuel oil or diesel in many static applications including boilers, furnaces, engines, and turbines for electricity generation. There are also a range of chemicals that can be extracted or derived from pyrolysis oils including food flavorings, resins, agrochemicals, fertilizers, and emission control agents [1]. Considering their wide range of potential applications, understanding the molecular composition of the pyrolysis oils and upgraded products is highly desirable because it allows the determination of molecular-product property relations and the molecular processes taking place that are crucial information for the upgrading of the pyrolysis oils. It is also important for determining the changes occurring in the pyrolysis oils during handling, storage, and utilization [2]. Furthermore, the environmental impacts naturally depend on the nature of the chemical constituents; for example, polyaromatic hydrocarbons (PAH) and benzenes are less environmentally friendly than anhydrosugars such as levoglucosan.

Because the pyrolysis products are complex, highly oxygenated, and unstable, analysis of pyrolysis liquids is a challenge, and the direct application of standard methods known, for example, from petroleum liquid analysis is not possible. There are several publications on characterization of fast pyrolysis liquids [3-5], but they all agree that there is no standard pyrolysis oil; the properties of the liquid are strongly dependent on feedstock and production methods. In the course of the development of thermochemical biomass conversion processes, a large number of chemical analyses procedures have been developed and tested comprising fractionation, gas chromatography, liquid chromatography, and spectroscopic methods [6-9]. A comprehensive overview of chemical and physical characterization methods for biomass pyrolysis oils has been reported by Fagernas [10], and Milne et al. [5]. The chemical characterization of pyrolysis oils has generally been based on the solvent fractionation of the oils into different classes of chemical functionality [10 12]. In solvent extraction, the oils have been divided mainly 


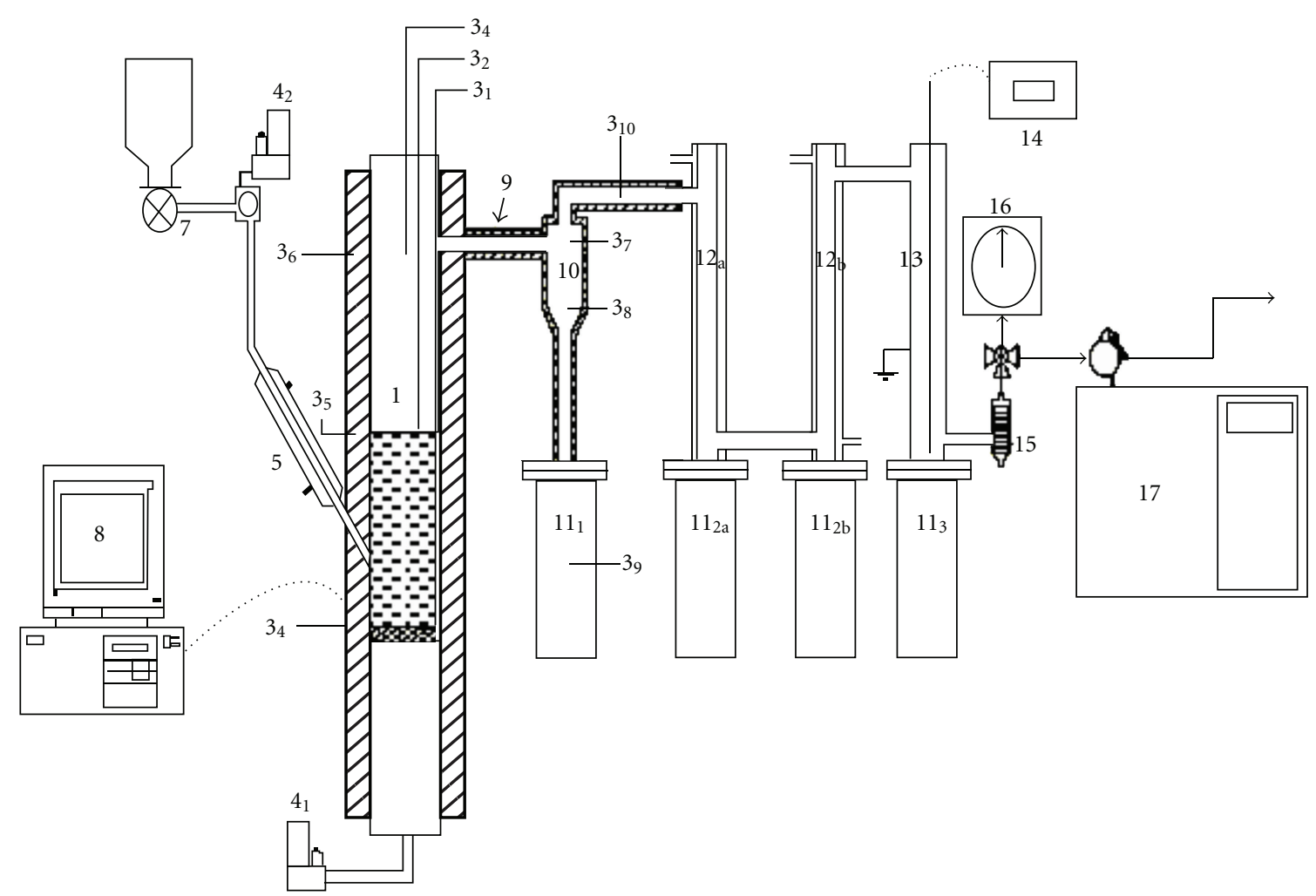

Figure 1: Schematic diagram of the fluidized bed reactor unit (1-fluidized bed, 3-thermocouple, 4-mass flow controller, 5- jacketed aircooled feeder tube, 6-hopper, 7-screw feeder, 8-computer, 9-heating tape, 10-hot gas filter, 11-reservoir, 12-condenser, 13-ESP, 14-AC power supply, 15-filter, 16-Wet gas meter, 17-Gas Chromatograph).

into acidic, phenolics, basic, hydrocarbons, and aqueous fractions. Gas chromatography-mass spectrometry (GC/MS) has been the technique most widely used in the analyses of the fractions separated using the solvent fractionation method $[12,13]$.

The chemistry of pyrolysis oils mostly depends on the type of biomass used and on the process severity (temperature, residence time, and heating rate profiles) and can be manipulated by changing the thermal conditions of the process or by conducting pyrolysis in the presence of catalysts [14-19]. In this paper, we report the column fractionation and GC/MS analysis of pine wood catalytic pyrolysis oils.

\section{Experimental}

2.1. Materials and Methods. The biomass used in the experiments was Pinus radiata (pine wood) that was harvested by the Virginia Tech Forestry club during the summer of 2008. The pine logs were ground with disc mill and dried to equilibrium moisture content and then stored in barrels until the time of pyrolysis. Subsamples of the disc milled wood samples were ground in a Wiley mill to pass a $1-\mathrm{mm}$ screen, sieved to $-20 /+80$ mesh size, and oven dried to less than $10 \%$ moisture content. The oil was produced using fluidized bed pyrolysis technology. Two different commercial HZSM-5 catalysts obtained from BASF (BASF Inc, Florham, NJ) and
Sud-Chemie (Sud-Chemie Inc, Louisville, KY) were used in these studies.

Pine wood samples were studied under four different pyrolysis conditions:

(1) pine wood pyrolyzed on silica sand (sand),

(2) pine wood pyrolyzed on BASF HZSM-5 catalyst (Untr/BASF),

(3) sodium hydroxide treated pine wood and pyrolyzed on BASF HZSM-5 catalyst (Treat/BASF),

(4) sodium hydroxide treated pine wood and pyrolyzed on Sudchem HZSM-5 catalyst (treat/Sudchem).

The sodium hydroxide treatment was performed as follows: $\mathrm{NaOH}_{(\mathrm{s})}(1 \mathrm{wt} \%)$ was dissolved in deionized water and the solution was added to the wood sample, mixed thoroughly, and then oven dried at $105^{\circ} \mathrm{C}$.

2.2. Pyrolysis of Pine Wood. The fast pyrolysis was carried out in a bench-scale fluidized bed reactor unit located at the Bioresource Engineering Laboratory, Virginia Tech, USA (Figure 1). The unit is comprised of a K-Tron volumetric feeder, $50 \mathrm{~mm}$ bubbling fluidized bed reactor equipped with a $100 \mu \mathrm{m}$ porous metal gas distributor, hot gas filter, two chilled water condensers, an electrostatic precipitator, and a glass bead-packed column. The reactor was externally heated with a three-zone electric furnace (Thermcraft, 
TABLE 1: Yield of products from catalytic and noncatalytic pyrolysis of pine wood.

\begin{tabular}{lcccc}
\hline Sample & Total liquid (\%) & Char $(\%)$ & Gases $(\%)$ & Oil pH \\
\hline Sand & 62.1 & 8.31 & 14.20 & 2.90 \\
Untr/BASF & 39.37 & 25.02 & 35.61 & 3.68 \\
Treat/BASF & 36.53 & 16.16 & 47.31 & 3.58 \\
Treat/Sudchem & 43.3 & 35.1 & 21.5 & 3.30 \\
\hline
\end{tabular}

Winston-Salem, NC, USA). $100 \mathrm{~g}$ of silica sand was used as the fluidizing medium, and the bed was fluidized with nitrogen $(12 \mathrm{~L} / \mathrm{min})$. The silica sand particles had a surface mean diameter of $355 \mu \mathrm{m}$. The static bed height of $31 \mathrm{~mm}$ expanded to $40 \mathrm{~mm}$ when it was fully fluidized. $300 \mathrm{~g}$ of each pine wood sample was pyrolyzed at $450^{\circ} \mathrm{C}$. They were fed at a rate of $300-330 \mathrm{~g} / \mathrm{h}$ from a feed hopper using a screw feeder to convey it into an entrainment zone. $6 \mathrm{~L} / \mathrm{min}$ of nitrogen gas was used to entrain the feed through a jacketed air-cooled feeder tube into the fluidized bed. The reactor temperature, feed rate, and gas flow rate were kept constant for each run. During pyrolysis, the mixture of char, gases, and vapors that exited the reactor was separated by a hot gas filter maintained at $400^{\circ} \mathrm{C}$. The separated gases and vapors were then passed through two condensers connected in series. The condensers were maintained at $-8^{\circ} \mathrm{C}$ with a $50 / 50$ cooling mixture of ethylene glycol and water from an 18-liter A82 refrigerated circulating bath (Haake, Karlsruhe, Germany). Any condensable gases and aerosols that escaped from the condenser were captured by an electrostatic precipitator (ESP) kept at $16-20 \mathrm{kV}$ and a packed column of glass beads. For the catalytic pyrolysis, the sand was replaced with $150 \mathrm{~g}$ of catalyst.

2.3. Column Fractionation of Pyrolysis Oil. The pyrolysis oils were fractionated to gain more insights into their composition. The oil sample (110-120 mg) dissolved in methanol $(2-3 \mathrm{~mL})$ was added to $1.5 \mathrm{~g}$ of silica gel $(0.105-$ $0.2 \mathrm{~mm}$ particle size and $60 \AA$ average pore size) in a porcelain crucible. The silica/oil mixture was heated to $40^{\circ} \mathrm{C}$ on a hot plate under a fumehood to evaporate the excess solvent. The oil-coated silica gel was added to the top of a glass column $(21.5 \mathrm{~cm}$ height $\times 2.2 \mathrm{~cm}$ i.d. $)$ packed with silica gel/hexane to about $2 / 3$ of the column height. The pyrolysis oil was eluted sequentially with the following solvents: hexane $(100 \mathrm{~mL})$, toluene $(50 \mathrm{~mL})$, ethyl acetate $(150 \mathrm{~mL})$, and methanol $(100 \mathrm{~mL})$ to produce aliphatics, aromatics, phenols, and more polar subfractions, respectively. Each fraction was evaporated using a rotary vacuum evaporator at $40-60^{\circ} \mathrm{C}$, depending on the solvent. Finally, the hexane, toluene, and ethyl acetate fractions were analyzed by GC-MS. After the evaporation of solvent from the methanol fraction, the solid residue was washed with distilled water in order to obtain water-soluble and water-insoluble fractions. The water-soluble fraction was analyzed for levoglucosan content by HPLC [20]. The water insolubles were not analyzed.

2.4. GC-MS Analysis of Pyrolysis Oils. The separation was made on a HP-5 fused silica capillary column $(30 \mathrm{~m}$,
$0.25 \mu \mathrm{m}, 0.32 \mathrm{~mm}$ i.d.) using a HP $5890 \mathrm{Gas}$ Chromatograph (Agilent Technologies Inc., Wilmington, DE). The GC oven temperature was held at $50^{\circ} \mathrm{C}$ for $1 \mathrm{~min}$ then ramped to $270^{\circ} \mathrm{C}$ at $7^{\circ} \mathrm{C} / \mathrm{min}$ and then held at this temperature for $10 \mathrm{~min}$. The injector temperature was $190^{\circ} \mathrm{C}$ with $50: 1$ split ratio. A $1 \mathrm{~mL} / \mathrm{min}$ helium was used as the carrier gas. The analytes were prepared as $6 \%$ solutions in methanol. The end of the column was directly introduced into the ion source of the Hewlett-Packard model 5972 series mass selective detector (MSD) operated with electron impact ionization at $70 \mathrm{eV}$. The data acquisition system used was G1034 C Chemstation software with a NBS library.

\section{Results and Discussion}

3.1. Pyrolysis Product Yields. The yields of pyrolysis products ranged from $36 \mathrm{wt} \%$ to $62 \mathrm{wt} \%$ depending on the catalyst and treatment conditions (Table 1). The pyrolysis with sand produced the highest yield of oil $(62.1 \mathrm{wt} \%)$ with a low $\mathrm{pH}$ of 2.90. The catalytic pyrolysis of untreated biomass using BASF decreased the yield of oil to $39 \mathrm{wt} \%$ because some of the carbohydrate decomposition products were gasified, and consequently gas yield was very high [21]. The treatment of the pine wood with sodium hydroxide solution before the catalytic pyrolysis also decreased the yield of oil and increased the gas yield considerably. The effect of sodium hydroxide solution treatment was different for the two catalysts. The treated pine wood pyrolyzed on BASF catalyst produced less char than a similar sample pyrolyzed on Sudchem catalyst. The differences in char yield were attributed to the differences in the acidity of the two catalysts and perhaps the influence of the sodium ions on the catalysts. Sodium is known to enhance sintering $[22,23]$ which will reduce the surface area of the catalyst and therefore its activity. The more active catalyst tends to produce more char than the less active catalyst; however surface areas of the catalysts were not measured to verify this assertion. The $\mathrm{pHs}$ of the catalytic pyrolysis oils were relatively higher than that of the pyrolysis oil from the sand pyrolysis. The differences in the $\mathrm{pHs}$ were also attributed to the gasification of some of the acidic components during the catalytic pyrolysis [21].

3.2. Fractionation of Pyrolysis Oils. After column fractionation of the pyrolysis oils and solvent evaporation, the fractions were weighed, and mass balances for the samples were determined. The mass balances showed 95-97\% recovery from the fractionation process (Table 2). The loss of material was attributed to a combination of high-mass material retained on the silica gel and low-mass material 
TABLE 2: Column fractionation of various pyrolysis oils (wt\%).

\begin{tabular}{lccccc}
\hline Sample & Hexane & Toluene & Ethyl acetate & Methanol & Levoglucosan* (water sol/total) \\
\hline Sand & 2.6 & 1.3 & 54.5 & 26.6 & 6.7 \\
Untr/BASF & 1.8 & 1.8 & 54.0 & 14.5 & 4.3 \\
Treat/BASF & 2.2 & 1.8 & 61.4 & 6.6 & 2.4 \\
Treat/Sudchem & 1.4 & 2.8 & 58.0 & 11.3 & 4.4 \\
\hline
\end{tabular}

* Mass (mg). (Sand: untreated wood pyrolyzed on silica sand; Untr/BASF: untreated wood pyrolyzed on BASF catalyst; treat/BASF: wood treated with sodium hydroxide and pyrolyzed on BASF catalyst; treat/Sudchem: wood treated with sodium hydroxide and pyrolyzed on Sudchem catalyst).

lost during the evaporation of the solvent because the highly volatile components were not detected in the GC/MS analysis of the samples. The solvent fractionation of the pyrolysis oils showed that the distribution of various oil fractions was affected by the catalyst type, the sodium hydroxide treatment, and the combination of both methods (Table 2). All samples had low hexane and toluene soluble fractions suggesting low hydrocarbon content. In all cases, the total hydrocarbon content (hexane + toluene fractions) was less than $5 \mathrm{wt} \%$ of the pyrolysis oil. The ethyl acetate fraction was the most abundant, constituting from 54 to $61 \mathrm{wt} \%$ of the pyrolysis oils. This fraction was mostly phenolic compounds and varied with the pyrolysis process conditions and biomass pretreatment. The pyrolysis on BASF catalyst of sodium hydroxide-treated sample (Treat/BASF) produced the highest yield of ethyl acetate fraction $(61 \mathrm{wt} \%)$, while the untreated biomass sample pyrolyzed on BASF catalyst (Untr/BASF) produced the lowest ethyl acetate fraction (54 wt\%).

The methanol fraction showed the widest yield variation ranging from $6.6 \mathrm{wt} \%$ to $26 \mathrm{wt} \%$ depending on the catalyst and biomass pretreatment. The variation in levoglucosan content of the pyrolysis oils followed a similar trend as the methanol fraction. The pine wood without sodium hydroxide treatment pyrolyzed on sand (Sand) produced the highest fraction of methanol soluble $(26 \mathrm{wt} \%)$ and subsequently the highest levoglucosan content (6.7 wt\%), while the Treat/BASF produced the lowest methanol soluble fraction $(6.6 \mathrm{wt} \%)$ and also the lowest levoglucosan yield. It is known that thermal decomposition of cellulose proceeds through two major pathways depending on the presence or absence of inorganic salts $[24,25]$. In the presence of potassium or sodium ions, levoglucosan production is strongly suppressed, and hydroxyacetaldehyde production is favored, whereas neutralization or treatment of biomass with mineral acids promotes the production of levoglucosan $[25,26]$. In the current studies, because the wood was treated with sodium hydroxide, there were obviously excess sodium ions that suppressed the formation of levoglucosan.

The methanol fraction and levoglucosan content of the pyrolysis oil produced from the Untr/BASF were lower than those of the Sand sample. This clearly suggested that solid acid effect was different from mineral acid effect on the biomass pyrolysis products. When biomass is treated with mineral acids such as sulfuric acid, the levoglucosan content is usually increased $[25,26]$ and since levoglucosan content is linearly correlated with the methanol fraction, this suggests that mineral acid treatment will increase the methanol fraction of the pyrolysis oils. The BASF catalyst probably degraded the levoglucosan molecules because they are small enough to enter the active sites of the catalyst. These results are in agreement with our previous studies on the fractional catalytic pyrolysis of hybrid poplar wood [21].

The fraction of methanol soluble was also strongly influenced by the catalytic reaction. The two catalysts reduced the fraction of methanol soluble whether the wood was treated or untreated with sodium hydroxide. The methanol soluble is generally carbohydrate decomposition products which are normally low molecular weight materials. Our previous studies showed that HZSM-5 catalysts in general tend to gasify these low molecular weight carbohydrate decomposition products because they are capable of penetrating the catalyst to the active site [27]. The sodium hydroxide treatment together with the catalyst appeared to reduce the methanol soluble fraction. The Untr/BASF reduced the methanol fraction by $45 \%$ relative to the Sand while the Treat/BASF reduced the methanol fraction by $75 \%$. This also suggests that the carbohydrate degradation products generated through the hydroxyacetaldehyde pathway were perhaps more easily degraded by the BASF catalyst.

The combination of the Sudchem catalyst and the sodium hydroxide treatment (Treat/Sudchem) reduced the methanol fraction by $57 \%$ compared to $75 \%$ by the Treat/ BASF on a similar substrate. The differences in the catalyst performances could be attributed to differences in either their acidities or surface areas.

3.3. Identification of the Major Components in the Pyrolysis Oils. The GC-MS analyses of the pyrolysis oil fractions revealed a large number of compounds. To determine the major compounds in the oil samples, a selection procedure was developed. In the first step, the absolute peak area of the solvent was eliminated from the total peak area. Next, the peak areas of all the compounds were summed up and converted to their relative area contribution (100\%) by using Microsoft Excel software. The first selection of compounds was performed by setting a minimum relative peak area greater than $0.3 \%$. This method was used to select the major constituents of the oil samples as shown in Tables 3(a), $3(\mathrm{~b})$, and 3(c). In this method a universal detector response was assumed. Although this assumption is not valid for the determination of absolute concentrations, it is useful for the determination of the relative concentrations of the individual compounds [7]. 
TABLE 3: (a) chemical composition of hexane fraction of pyrolysis oils (\% area). (b) chemical composition of toluene fraction of pyrolysis oils (\% area). (c) chemical composition of ethyl acetate fraction of pyrolysis oils (\% area).

(a)

\begin{tabular}{lcccc}
\hline Major components & Sand & Untr/BASF & Treat/BASF & Treat/SudChem \\
\hline (1) Benzene, 1-methyl-2-cyclopropen-1- & ND & ND & 3.52 & ND \\
(2) 1H-Indene, 1-methyl & ND & ND & 3.47 & ND \\
(3) Naphthalene & ND & ND & 4.74 & ND \\
(4) 1H-Indene, 1,1-dimethyl & ND & 2.71 & 8.38 & ND \\
(5) Naphthalene, 2-methyl & ND & ND & 21.63 & 8.21 \\
(6) Naphthalene, 1-ethyl & ND & 2.13 & 3.87 & ND \\
(7) 1-Tridecene & ND & 9.12 & 20.13 & 4.07 \\
(8) Naphthalene, 2,6-dimethyl & ND & ND & ND & 14.99 \\
(9) Naphthalene, 2,3-dimethyl & ND & 2.13 & ND & 4.95 \\
(10) Naphthalene, 2-(1-methylethyl) & ND & 3.61 & 4.51 & 5.02 \\
(11) 1-Dodecene & ND & 0 & 0 & 7.26 \\
(12) Cyclopropane, octyl & ND & 2.37 & ND & 4.68 \\
(13) 9-Octadecene, (E) & ND & 18.51 & ND & 4.34 \\
(14) Bis(2-ethylhexyl)phthalate & ND & 46.20 & 4.75 \\
(15) Total long chain aliphatics & 99.5 & & 4.35 \\
\hline
\end{tabular}

ND: not detected.

(b)

\begin{tabular}{|c|c|c|c|c|}
\hline Main components & Sand & Untrt/BASF & Treat/BASF & Treat/SudChem \\
\hline (1) Cyclohexane, ethyl & ND & ND & 6.13 & ND \\
\hline (2) Ethylbenzene & 12.35 & 11.59 & 14.71 & 13.93 \\
\hline (3) Benzene, 1,3-dimethyl & 9.78 & ND & 10.29 & 11.23 \\
\hline (4) Benzofuran, 2-methyl & ND & ND & ND & 5.61 \\
\hline (5) Cinnoline, 1,2-dihydro-2-methyl & ND & ND & ND & 12.06 \\
\hline (6) $1 \mathrm{H}$-indene, 1,1-dimethyl & ND & ND & ND & 6.65 \\
\hline (7) Ethanone, 1-[4-(1-methylethenyl)phenyl] & ND & ND & ND & 5.41 \\
\hline (8) Benzene, 1,4-bis-(1-methylethenyl) & ND & ND & ND & 2.91 \\
\hline (9) Benzene, hexamethyl & ND & ND & ND & 5.61 \\
\hline (10) Naphthalene, 1,4,6-trimethyl & ND & ND & ND & 5.82 \\
\hline (11) Benzene, 1-methyl-4-(1-methylethyl) & ND & ND & ND & 4.99 \\
\hline (12) Phenanthrene, 2,4,5,7-tetramethyl & ND & 3.12 & 20.83 & 11.02 \\
\hline (13) Bis(2-ethylhexyl)phthalate & 71.18 & 32.13 & 48.04 & 3.12 \\
\hline (14) Phthalate derivatives & ND & 43.33 & ND & ND \\
\hline
\end{tabular}

ND: not detected.

(c)

\begin{tabular}{|c|c|c|c|c|}
\hline Main components & Sand & Untr/BASF & Treat/BASF & Treat/SudChem \\
\hline (1) Propanal & 0.25 & ND & ND & ND \\
\hline (2) 2-Cyclopentene-1-one & ND & ND & 0.33 & 0.86 \\
\hline (3) 2-Cyclopentene-1-one, 2-methyl & ND & ND & 0.65 & 0.73 \\
\hline (4) $2(5 \mathrm{H})$-furanone & 1.68 & 1.09 & ND & ND \\
\hline (5) 2-Hydroxycyclopent-2-en-1-one & 1.06 & ND & 0.16 & ND \\
\hline (6) Benzaldehyde & ND & ND & 0.27 & 0.31 \\
\hline (7) 1,2-Pentadiene & $\mathrm{ND}$ & ND & 0.22 & 0.70 \\
\hline (8) 2(5H)-Furanone, 3-methyl & 0.69 & 0.36 & ND & ND \\
\hline (9) Phenol & 1.06 & 0.9 & 1.95 & 3.04 \\
\hline (10) 1,2-Cyclopentanedione, 3-methyl & 3.26 & 2.41 & ND & ND \\
\hline (11) 2-Cyclopentene-1-one, 2-hydroxy-3-methyl & ND & ND & ND & 1.95 \\
\hline (12) 2,3-dimethylcyclopent-2-en-1-one & ND & ND & 0.56 & ND \\
\hline (13) Phenol, 2-methyl & 1.49 & 0.68 & 1.66 & 2.32 \\
\hline (14) Phenol, 4-methyl & ND & 1.8 & ND & ND \\
\hline
\end{tabular}


(c) Continued.

\begin{tabular}{|c|c|c|c|c|}
\hline Main components & Sand & Untr/BASF & Treat/BASF & Treat/SudChem \\
\hline (15) Phenol, 3-methyl & 2.68 & ND & 3.10 & 4.61 \\
\hline (16) Phenol, 2-methoxy & 2.62 & 1.19 & 4.93 & 4.22 \\
\hline (17) Phenol, 2,3-dimethyl & ND & ND & 0.49 & 0.48 \\
\hline (18) Phenol, 2-ethyl & ND & ND & 0.39 & 0.43 \\
\hline (19) Phenol, 3,5-dimethyl & 1.53 & 0.90 & 2.98 & 3.31 \\
\hline (20) Phenol, 4-ethyl & 1.38 & 1.26 & 1.01 & 1.14 \\
\hline (21) Phenol, 3-ethyl & ND & ND & 1.18 & 1.39 \\
\hline (22) Phenol, 2,4-dimethyl & ND & ND & 0.31 & 0.41 \\
\hline (23) Phenol, 2-methoxy-4-methyl & 3.61 & 1.47 & 0.30 & 0.73 \\
\hline (24) Benzene, 1,4-dimethoxy & ND & ND & 2.72 & 2.32 \\
\hline (25) Phenol, 3,4-dimethyl & ND & ND & 0.35 & 0.68 \\
\hline (26) Phenol, 2,4,6-trimethyl & ND & ND & 0.17 & ND \\
\hline (27) 1,2-Benzenediol & 9.33 & 8.41 & 6.53 & 7.69 \\
\hline (28) Benzofuran, 2,3-dihydro & ND & 2.31 & 0.92 & 1.48 \\
\hline (29) Phenol, 2,3,5-trimethyl & ND & ND & 0.22 & ND \\
\hline (30) Phenol, 2-ethyl-6-methyl & ND & 0.84 & 0.26 & 0.65 \\
\hline (31) Phenol, 2-(1-methylethyl) & 0.84 & ND & ND & ND \\
\hline (32) Phenol, 4-ethyl-3-methyl & ND & 0.47 & 1.27 & ND \\
\hline (33) Phenol, 3,4,5-trimethyl & 1.69 & ND & ND & 0.55 \\
\hline (34) 1,2-Benzenediol, 3-methyl & 2.29 & 2.06 & 2.65 & 3.55 \\
\hline (35) Phenol, 2,3,6-trimethyl & ND & ND & ND & 0.38 \\
\hline (36) Phenol, 4-ethyl-2-methoxy & 2.02 & 1.30 & 3.94 & 2.52 \\
\hline (37) $1 \mathrm{H}$-Indene-1-one, 2,3-dihydro & ND & ND & 0.40 & 0.43 \\
\hline (38) 1,2-Benzenediol, 4-methyl & 10.26 & 7.83 & 4.84 & 6.80 \\
\hline (39) 4-Hydroxy-2-methylacetophenone & ND & 2.13 & ND & ND \\
\hline (40) Ethanone, 1-(3-methoxyphenyl) & 2.26 & ND & 4.79 & 3.10 \\
\hline (41) 1H-Indene-5-ol, 2,3-dihydro & ND & 0.60 & 0.86 & 1.18 \\
\hline (42) Phenol, 4-(2-propenyl) & 1.48 & ND & ND & ND \\
\hline (43) 1,3-Benzenediol, 4-ethyl & ND & ND & 0.4292404 & 0.59727 \\
\hline (44) Eugenol & 4.34 & ND & ND & ND \\
\hline (45) Phenol, 2-methoxy-4-propyl & 1.06 & 0.76 & ND & ND \\
\hline (46) Benzaldehyde, 4-hydroxy & ND & 0.84 & ND & ND \\
\hline (47) 1,2-Benzenediol, 4,5-dimethyl & 6.07 & 3.28 & 0.42 & 0.61 \\
\hline (48) Vanillin & 4.70 & 5.31 & 3.38 & 2.70 \\
\hline (49) Phenol, 2-methoxy-4-(1-propenyl) & 6.45 & 1.47 & 6.32 & 4.72 \\
\hline (50) Ethanone, 1-(4-hydroxy-3-methoxyphenyl) & 2.86 & 1.98 & 1.26 & 0.95 \\
\hline (51) 1-Naphthalenol & ND & ND & 0.47 & 0.47 \\
\hline (52) 2-Naphthalenol & ND & 0.7 & 0.60 & 0.55 \\
\hline (53) 1-Naphthalenol, 2-methyl & ND & ND & 0.47 & 0.53 \\
\hline (54) Benzeneacetic acid, 4-hydroxy-3-methyl & 1.55 & ND & 1.05 & ND \\
\hline (55) Bis(2-ethylhexyl)Phthalate & 0.77 & 19.77 & 1.98 & 4.10 \\
\hline
\end{tabular}

ND: not detected.

The compounds identified in the hexane, toluene, and ethyl acetate fractions of the pyrolysis oil samples constituted 93-95\%, 88-99\%, and 67-79\%, respectively, of the total area composition. Approximately 80 compounds were identified by a probability match $>80$ using the GC-MS software library (Tables 3(a), 3(b), and 3(c)). The identification of compounds by their mass spectrum was seriously hampered by coelution of some peaks. However, several specific phenolic compounds such as guaiacols (e.g., methoxy(methyl)-phenols) and syringols (e.g., dimethoxy-phenols) were identified with high probabilities.

Using the above procedure the differences in the relative concentrations of the major chemical compounds in the pyrolysis oils were shown. Levoglucosan, furaldehyde, furanone, and other aldehydes and ketones from sugar degradations were identified. Other major compounds identified in 
the pyrolysis oils were guaiacols/syringols (methoxyphenols) and benzenediols.

\subsubsection{Composition of Hexane Soluble Fraction of Pyrolysis Oils.} The relative concentrations of the hexane soluble fractions of the pyrolysis oils are shown in Table 3(a) and Figure 2. The hexane fractions consisted of benzene derivatives, long chain aliphatic hydrocarbons (LCA), phthalate derivatives, and polycyclic hydrocarbons (PH). Clearly, both the catalyst and the treatment of the biomass affected the composition of these fractions. The untreated wood pyrolyzed on the sand contained $99.5 \%$ LCA. This LCA probably derived from the extractives content of the wood since pine is known to contain fats and waxes [28], which could decarboxylate to produce LCA.

The LCA content of Untr/BASF pyrolysis oil was 50\% lower than that from the Sand, which suggested that some of the fats and waxes (LCA precursors) reacted on the BASF catalyst to produce other compounds. Whereas $\mathrm{PH}$ and phthalate derivatives were very low or nonexistent in the hexane extract of the Sand oil, significant fractions were detected in the Untr/BASF pyrolysis oils suggesting that some of these products derived from the catalytic conversion of LCA precursors to $\mathrm{PH}$ such as naphthalene derivatives (15\%) and phthalate derivatives (25\%). These results were not surprising because HZSM-5 catalysts are known to promote the formation of aromatic compounds through the cyclization of long chain aliphatic compounds [29]. The BASF catalyst had a higher selectivity for phthalate derivatives compared to the polycyclic hydrocarbons.

The treatment of the wood with sodium hydroxide before pyrolysis on the BASF catalyst (Treat/BASF) changed the composition of hexane fraction drastically. The LCA content was reduced to almost zero, and the LCA precursor appeared to be converted to two major compounds $\mathrm{PH}$ $(>60 \%)$ and phthalate derivatives $(<20 \%)$. Under these conditions, the catalyst had a higher selectivity for PHs such as 2-methyl and 2,6-dimethyl naphthalenes compared to phthalate derivatives. The selectivity for phthalate derivatives was much lower than that for Untr/BASF. This suggests that the sodium hydroxide treatment has effect on the BASF active site responsible for the formation of the phthalate derivatives. In addition to these two major compounds, several other aromatic compounds were produced (Figure 2 and Table 3(a)).

In contrast to the Treat/BASF, the Treat/Sudchem contained over $60 \%$ LCA and about $30 \% \mathrm{PH}$, and virtually no phthalate derivatives were formed (Figure 3). The sodium hydroxide treatment of the feed appeared to deactivate the sites responsible for the production of the phthalate derivatives in both catalysts, but it is stronger in the Sudchem compared to the BASF. However, the site for the $\mathrm{PH}$ formation appeared to be positively affected in the case of the BASF catalyst and less so in the Sudchem. It is clear from Table 3(a) and Figure 2 that although both catalysts are $\mathrm{H}-\mathrm{ZSM}-5$, there are major differences in their product selectivity and distribution which could be probably due to differences in acidity and surface areas.

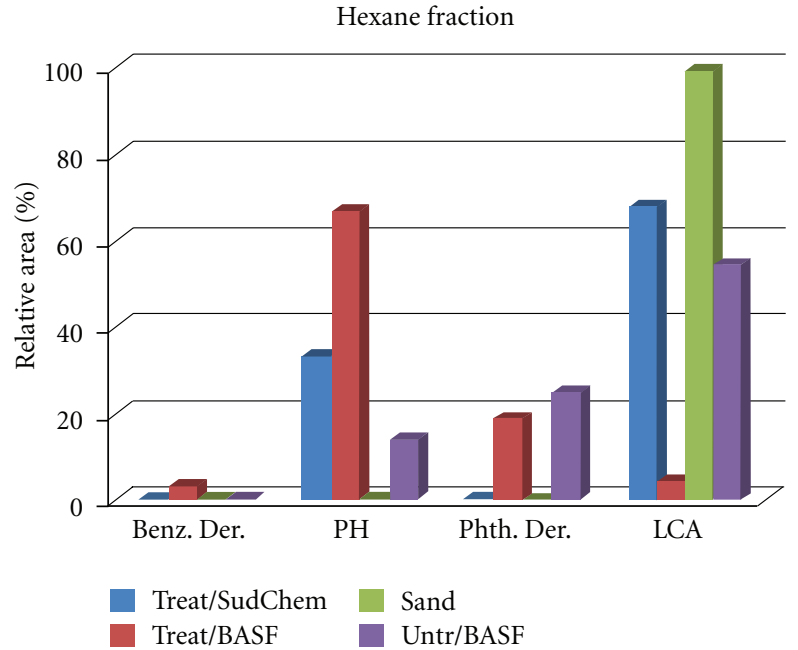

FIgURE 2: Relative distribution of major compounds identified in the hexane fraction of the pyrolysis oils. (Benzene der.: benzene derivatives; PH: polycyclic hydrocarbons; Phth. Der.: phthalate derivatives; LCA: long chain aliphatic hydrocarbons).

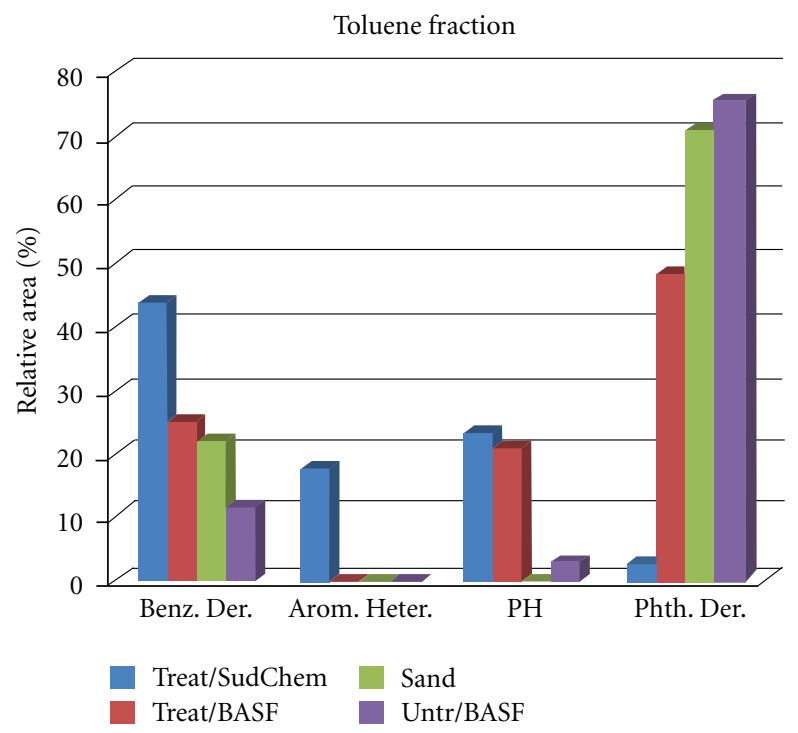

FIgURE 3: Relative distribution of major compounds in the toluene fraction of pyrolysis oils. (Benz. Der.: benzene derivatives; Arom. Heter.: aromatic heterocyclic compounds; PH: polycyclic hydrocarbons; Phth. Der.: phthalate derivatives).

3.3.2. Composition of Toluene Soluble Fraction of Pyrolysis Oils. The composition of the toluene fraction is shown in Table 3(b) and Figure 3, which is predominantly phthalate and benzene derivatives. About fourteen major compounds were identified, but the major compounds were phthalate derivatives and smaller fractions of benzene derivatives and polycyclic aromatics. The distribution of the products slate was strongly affected by the biomass treatment and the catalyst. For the sand pyrolysis oil, three major products were identified (ethylbenzene, 1,3-dimethylbenzene, and $\operatorname{Bis}(2-$ ethylhexyl)phthalate). For the Untr/BASF oil the major 
products were Bis(2-ethylhexyl)phthalate (32\%) and phthalate derivatives (43\%); additionally there were ethylbenzene and small fractions of phenanthrene. The composition of the toluene fraction from Treat/BASF oil sample was moderately high in phthalate derivatives and relatively low in $\mathrm{PH}$ and benzene derivatives (Table 3(b), and Figure 3). Ethyl cyclohexane and 1,3 dimethyl benzene were also present in the Treat/BASF toluene fraction, but there were aromatic heterocyclic compounds.

The toluene fraction of Treat/Sudchem contained a wider slate of compounds compared to the Treat/BASF. This catalyst showed very little product selectivity, and mass closure was only $75 \%$. The Treat/Sudchem was very low in phthalate derivatives but was relatively higher in benzene derivatives (Figure 3). Thus, it appeared that the sodium hydroxide treatment strongly deactivated the site responsible for the formation of phthalate derivatives which is agreement with the composition of the hexane fraction which also showed a similar trend.

3.3.3. Composition of Ethyl Acetate-Soluble Fraction of Pyrol$y$ sis Oils. The ethyl acetate soluble was the predominant fraction of the pyrolysis oils, but it was also the most complex (Table 3(c), Figure 4). It contained 55 major compounds compared to 15 for the other two fractions. The fraction was composed of ketones, aldehydes, phenols, and phthalates. Unlike hybrid poplar wood catalytic pyrolysis products where the lignin moiety was extensively demethoxylated/demethylated, there appeared to be very little demethoxylation of the lignin-derived compounds [21]. The catalysts did not appear to have any major effect on the selectivity of the products and neither did the sodium hydroxide treatment nor the combination of catalysts and sodium hydroxide treatment (Figure 4 ). The pore size of HZSM-5 is smaller than most lignin decomposition products [27], and therefore they are not capable of penetrating the catalyst to the active sites for reaction to take place. Thus, the trends in product distribution for the reaction on the sand pyrolysis medium were similar to those on the catalysts. However, quantitatively, the catalytic experiments had higher yields of ethyl acetate fraction than the noncatalytic pyrolysis experiments (Table 2 ).

\section{Conclusions}

The pretreatment of pine wood with sodium hydroxide solution and pyrolyzing on zeolite catalysts had significant influence on the distribution of the pyrolysis oil compounds. The relative composition of the oils may be obtained by summation of the relative areas of groups of compounds. In this way, determination of the relative change in molecular composition between the various oils is possible. With this approach, it was shown that the polycyclic hydrocarbons content of the pyrolysis oils increased under the catalytic pyrolysis conditions. The catalysts showed no selectivity for the phenolic components in the pyrolysis oils. The relative content of phenolic products was similar to those from the pyrolysis on the silica sand.

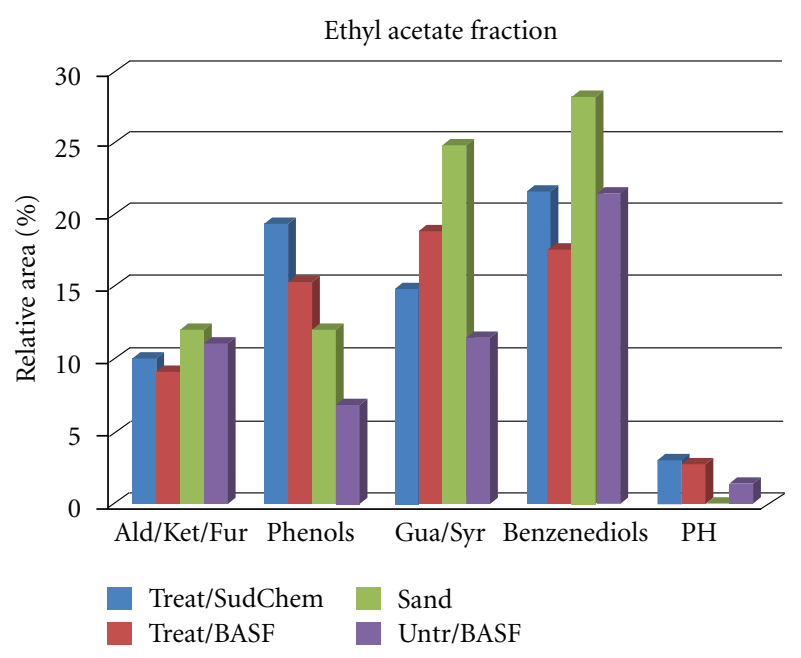

FIGURE 4: Relative distribution of major compounds in the ethyl acetate fraction of the pyrolysis oils. (Ald/Ket/Fur: aldehydes, ketones and furans; Phenols: phenolic compounds; Gua/Syr: Guaiacyl and syringyl-derived compounds; Benzenediols; $\mathrm{PH}$ : polycyclic hydrocarbons).

The production of LCA and selectivity of the catalysts towards phthalate derivatives were strongly influenced by the sodium hydroxide treatment of the wood before the pyrolysis. The GC/MS analysis thus allows us to obtain rapid and reliable insights into the molecular processes taking place during the catalysis process. This method can also be used screen catalysts to develop the catalytic pyrolysis technology. Furthermore, the pretreatment of the biomass can enhance the selectivity of the biomass pyrolysis products.

\section{Acknowledgment}

The USA Department of Energy Biomass Program is acknowledged for funding the research (contract no. DEFG36-08GO18214-1).

\section{References}

[1] S. Czernik and A. V. Bridgwater, "Overview of applications of biomass fast pyrolysis oil," Energy and Fuels, vol. 18, no. 2, pp. 590-598, 2004.

[2] A. V. Bridgwater, D. Meier, and D. Radlein, "An overview of fast pyrolysis of biomass," Organic Geochemistry, vol. 30, no. 12, pp. 1479-1493, 1999.

[3] V. A. Huffman and A. V. Bridgwater, The Characterization of Fast Pyrolysis Bio-Oils, Advances in Thermochemical Biomass Conversion, vol. 2, Blackie, London, UK, 1st edition, 1994.

[4] R. Maggi and B. Delmon, Characterization of Bio-Oils Produced By Pyrolysis, Advances in Thermochemical Biomass Conversion, vol. 2, Blackie, London, UK, 1st edition, 1994.

[5] T. Milne, F. Agblevor, M. Davis, S. Deutch, and D. Johnson, A Review of the Chemical Composition of Fast-Pyrolysis Oils From Biomass, Blackie, London, UK, 1997.

[6] L. Ingram, D. Mohan, M. Bricka et al., "Pyrolysis of wood and bark in an auger reactor: physical properties and chemical 
analysis of the produced bio-oils," Energy and Fuels, vol. 22, no. 1, pp. 614-625, 2008.

[7] J. H. Marsman, J. Wildschut, F. Mahfud, and H. J. Heeres, "Identification of components in fast pyrolysis oil and upgraded products by comprehensive two-dimensional gas chromatography and flame ionisation detection," Journal of Chromatography A, vol. 1150, no. 1-2, pp. 21-27, 2007.

[8] B. Scholze and D. Meier, "Characterization of the waterinsoluble fraction from pyrolysis oil (pyrolytic lignin). Part I. PY-GC/MS, FTIR, and functional groups," Journal of Analytical and Applied Pyrolysis, vol. 60, no. 1, pp. 41-54, 2001.

[9] E. Mészáros, E. Jakab, and G. Várhegyi, “TG/MS, Py-GC/MS and THM-GC/MS study of the composition and thermal behavior of extractive components of Robinia pseudoacacia," Journal of Analytical and Applied Pyrolysis, vol. 79, no. 1-2, pp. 61-70, 2007.

[10] L. Fagernas, Chemical and Physical Characterization of Biomass-Based Pyrolysis Oils. Literature Review, Technical Research Centre of Finland, Espoo, Finland, 1995.

[11] M. Garcia-Perez, A. Chaala, H. Pakdel, D. Kretschmer, and C. Roy, "Characterization of bio-oils in chemical families," Biomass and Bioenergy, vol. 31, no. 4, pp. 222-242, 2007.

[12] K. Sipilä, E. Kuoppala, L. Fagernäs, and A. Oasmaa, "Characterization of biomass-based flash pyrolysis oils," Biomass and Bioenergy, vol. 14, no. 2, pp. 103-113, 1998.

[13] K. L. Sobeih, M. Baron, and J. Gonzalez-Rodriguez, "Recent trends and developments in pyrolysis-gas chromatography," Journal of Chromatography A, vol. 1186, no. 1-2, pp. 51-66, 2008.

[14] S. Yaman, "Pyrolysis of biomass to produce fuels and chemical feedstocks," Energy Conversion and Management, vol. 45, no. 5, pp. 651-671, 2004.

[15] A. V. Bridgwater, "Catalysis in thermal biomass conversion," Applied Catalysis A, vol. 116, no. 1-2, pp. 5-47, 1994.

[16] J. Adam, M. Blazsó, E. Mészáros et al., "Pyrolysis of biomass in the presence of Al-MCM-41 type catalysts," Fuel, vol. 84, no. 12-13, pp. 1494-1502, 2005.

[17] J. F. Li, R. Yan, B. Xiao, X. L. Wang, and H. Yang, "Influence of temperature on the formation of oil from pyrolyzing palm oil wastes in a fixed bed reactor," Energy and Fuels, vol. 21, no. 4, pp. 2398-2407, 2007.

[18] M. Garcia-Perez, S. Wang, J. Shen, M. Rhodes, W. J. Lee, and C. Z. Li, "Effects of temperature on the formation of ligninderived oligomers during the fast pyrolysis of Mallee woody biomass," Energy and Fuels, vol. 22, no. 3, pp. 2022-2032, 2008.

[19] D. Mohan, C. U. Pittman, and P. H. Steele, "Pyrolysis of wood/biomass for bio-oil: a critical review," Energy and Fuels, vol. 20, no. 3, pp. 848-889, 2006.

[20] F. A. Agblevor, A. Murden, and B. R. Hames, "Improved method of analysis of biomass sugars using high-performance liquid chromatography," Biotechnology Letters, vol. 26, no. 15, pp. 1207-1210, 2004.

[21] F. A. Agblevor, S. Beis, N. O. Mante, and N. Abdoulmoumine, "Fractional catalytic pyrolysis of hybrid poplar wood," Industrial and Engineering Chemistry Research, vol. 49, no. 8, pp. 3533-3538, 2010.

[22] C. N. Satterfield, Heterogeneous Catalysis in Industrial Practice, McGraw-Hill, New York, NY, USA, 1981.

[23] J. Álvarez, S. Ordóñez, R. Rosal, H. Sastre, and F. V. Díez, “A new method for enhancing the performance of red mud as a hydrogenation catalyst," Applied Catalysis A, vol. 180, no. 1-2, pp. 399-409, 1999.

[24] S. Julien, E. Chornet, P. K. Tiwari, and R. P. Overend, "Vacuum pyrolysis of cellulose: fourier transform infrared characterization of solid residues, product distribution and correlations," Journal of Analytical and Applied Pyrolysis, vol. 19, pp. 81-104, 1991.

[25] J. Piskorz, D. Radlein, D. S. Scott, and S. Czernik, "Pretreatment of wood and cellulose for production of sugars by fast pyrolysis," Journal of Analytical and Applied Pyrolysis, vol. 16, no. 2, pp. 127-142, 1989.

[26] S. Julien, E. Chornet, and R. P. Overend, "Influence of acid pretreatment $\left(\mathrm{H}_{2} \mathrm{SO}_{4}, \mathrm{HCl}, \mathrm{HNO}_{3}\right)$ on reaction selectivity in the vacuum pyrolysis of cellulose," Journal of Analytical and Applied Pyrolysis, vol. 27, no. 1, pp. 25-43, 1993.

[27] F. W. Atadana, Catalaytic pyrolysis of cellulose, hemicellulose and lignin model compounds [M.S. thesis], Virginia Polytechnic Institute and State University, 2010.

[28] D. Fengel and G. Wegener, Wood Chemistry, Ultrastructure, Reaction, Walter De Gruyter, New York, NY, USA, 1989.

[29] P. A. Horne and P. T. Williams, "The effect of zeolite ZSM-5 catalyst deactivation during the upgrading of biomass-derived pyrolysis vapours," Journal of Analytical and Applied Pyrolysis, vol. 34, no. 1, pp. 65-85, 1995. 

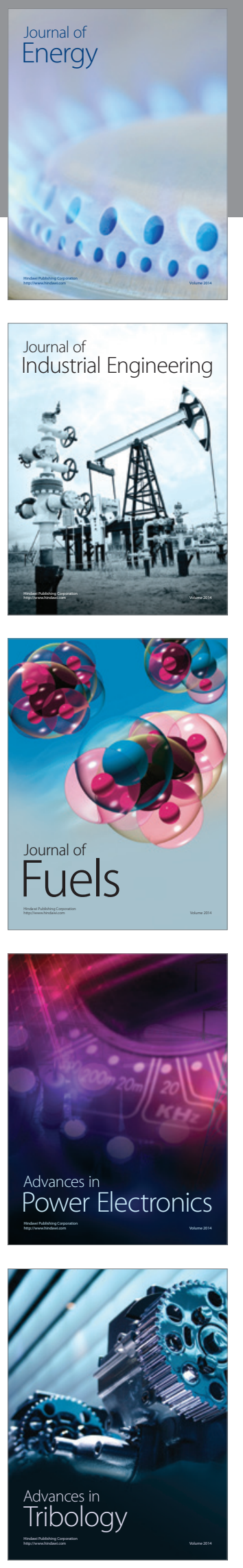
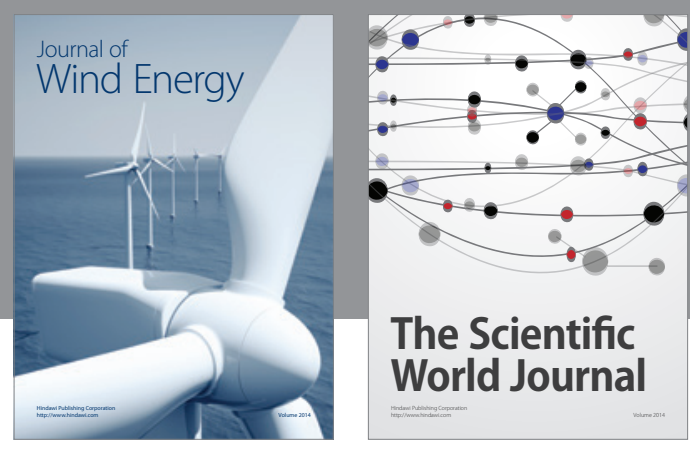

The Scientific World Journal

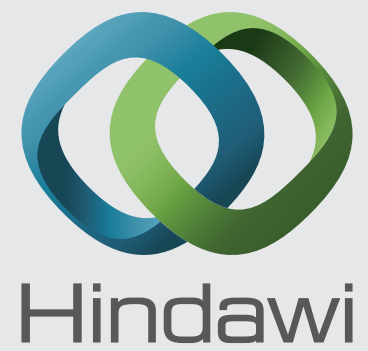

Submit your manuscripts at http://www.hindawi.com
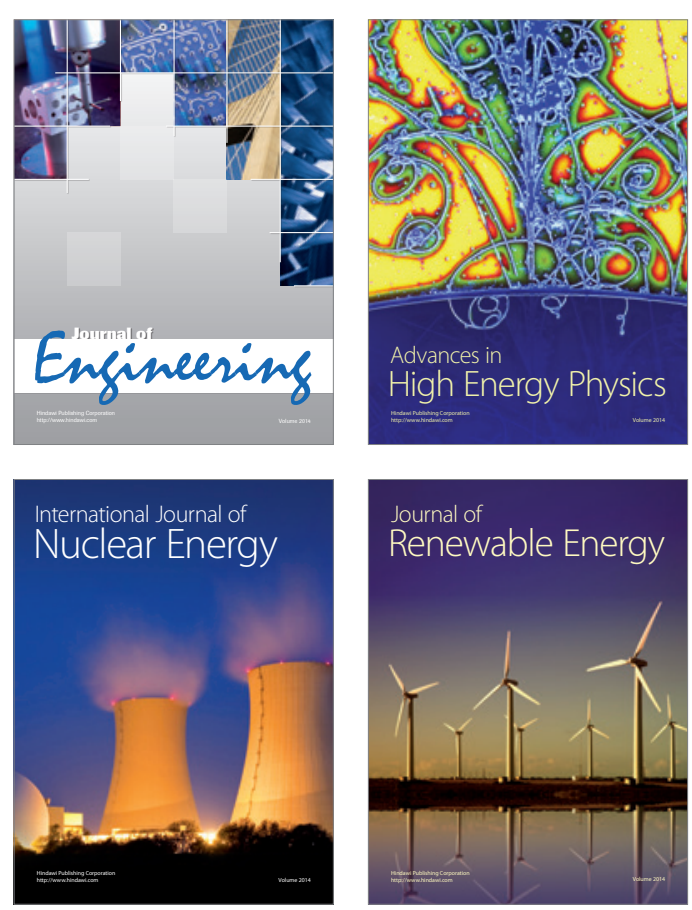

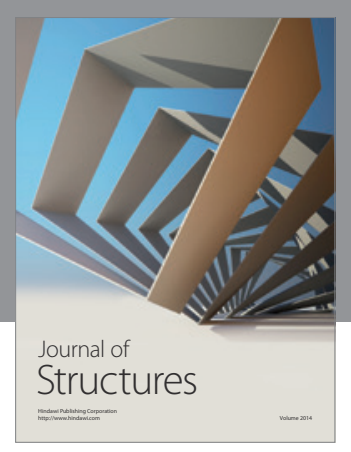

Rotating
Mechinery
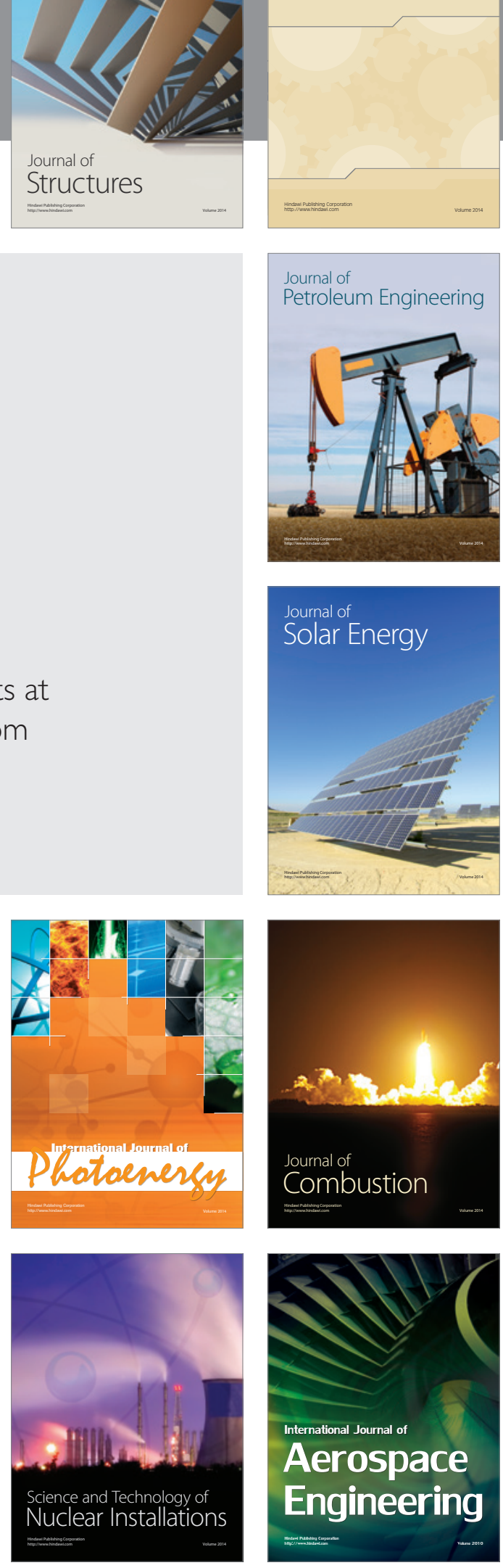